\title{
A population-based study on the association between urinary calculi and kidney cancer
}

\author{
Shiu-Dong Chung, MD; ${ }^{*+}$ Shih-Ping Liu, MD; Herng-Ching Lin, PhD ${ }^{\ddagger *}$
}

*Division of Urology, Department of Surgery, Far Eastern Memorial Hospital, Ban Ciao, Taipei, Taiwan; †Sleep Research Center, Taipei Medical University Hospital, Taipei, Taiwan; §Department of Urology, National Taiwan University Hospital and College of Medicine, Taipei, Taiwan; "School of Health Care Administration, Taipei Medical University, Taipei, Taiwan

Cite as: Can Urol Assoc J 2013;7(11-12):e716-21. http://dx.doi.org/10.5489/cuaj.366 Published online November 8, 2013.

\section{Abstract}

Background: Using a nationwide population-based dataset and case-control study design, we investigate the association between urinary calculi (UC) and kidney cancer (KC) in Taiwan.

Methods: The data for this case-control study were sourced from the Taiwan National Health Insurance program. The cases included 1308 incident patients pathologically diagnosed with KC. This study also used 6540 randomly selected subjects as controls. Conditional logistic regression was used to examine the associations between KC and patients previously diagnosed with UC.

Results: Of the sampled patients, 1262 (16.1\%) had previously been diagnosed with UC; 415 (31.7\% of the patients with KC) and 847 controls ( $13.0 \%$ of patients without KC). After adjusting for monthly income, geographic location, urbanization level, hypertension, diabetes, renal disease, obesity, cystic kidney disease, tobacco use disorder, and alcohol abuse, we found that patients with $\mathrm{KC}$ were likely to have been previously diagnosed with UC than controls (odds ratio [OR] 3.18, 95\% confidence interval [CI] 2.75-3.68, $p<0.001)$. In addition, the magnitude of the observed associations were stronger among females (females OR 3.59; 95\% $\mathrm{Cl} 2.87-4.48$ vs. males OR 2.93, 95\% Cl 2.42-3.55) and transitional cell carcinoma patients (transitional cell carcinoma, OR 3.96; 95\% Cl 3.23-4.86 vs. renal cell carcinoma OR 2.76, 95\% Cl 2.31-3.29). Conclusions: We conclude that there is an association between $\mathrm{KC}$ and prior $\cup C$, especially in females and patients with transitional cell carcinoma.

\section{Introduction}

Urinary calculi (UC) is a common genitourinary disorder that has had an increasing incidence over the past 100 years and is currently estimated to occur during in $10 \%$ to $15 \%$ of the global population. ${ }^{1-4}$ Therefore, it is important to understand what conditions may be associated with the many survivors of this low-mortality condition.
It has been proposed that UC may cause alterations in the local environment due to chronic irritation and, subsequently, may lead to urothelial proliferation and the development of malignant neoplasms, especially transitional cell carcinoma (TCC). Another type of cancer that may be associated with UC is renal cell carcinoma (RCC). TCC arises from the tissues of the renal pelvis and ureter, while RCC arises from the tumours of the renal parenchyma. ${ }^{5}$

Numerous case reports have been published suggesting a link between UC and TCC, but few case-control studies examine this association. ${ }^{5}$ Although earlier studies have suggested an non-statistically significant association between UC and TCC, ${ }^{6-8}$ Chow and colleagues reported significant excesses of renal pelvis/ureter cancer based on standardized incidence ratios. ${ }^{5}$

In addition, there is controversy surrounding the association between RCC and UC. Although several case-control studies have successfully reported a significant association between RCC and UC, ${ }^{9-12}$ Chow and colleagues were unable to detect this association and refuted previous findings. ${ }^{5}$

To clarify this issue, we investigate the association between $\mathrm{KC}$ and patients with previously diagnosed UC, after adjusting for socio-demographic and medical characteristics.

\section{Methods}

\section{Database}

The data for this case-control study were sourced from Taiwan National Health Insurance [NHI] program. Taiwan inaugurated its single-payer NHI program in 1995 to provide affordable, comprehensive, and easily accessible medical care for all its citizens. The Longitudinal Health Insurance Database (LHID) 2005, which is derived from Taiwan's Bureau of $\mathrm{NHI}$ records and maintained by the Taiwan National Health Research Institute, includes the registra- 
tion files and original claims data for the reimbursement of 1 million beneficiaries under the NHI program. The LDH2000 includes randomly sampled data from the 2000 Registry for Beneficiaries ( $n=23.72$ million) of the Taiwan NHI program. The annual release of the LHID2000 includes the following dimensions: contracted medical care institutions, health professionals in contracted medical care institutions, beds in contracted medical institutions, departments in contracted medical institutions, outpatient medical benefit claims, inpatient medical benefit claims, prescriptions and treatment procedures in ambulatory care, inpatient prescriptions and treatment procedures, prescriptions from contracted pharmacies, and detailed inpatient costs.

One study by Gau and colleagues has validated the validity of the LHID2000 and found no significant difference in the gender distribution between patients selected in the LHID2000 and all patients enrolled under the $\mathrm{NHI}$ program. ${ }^{13}$ Furthermore, prior studies have demonstrated the high validity of the data from the NHI program. ${ }^{14,15}$ The LHID2000 allows researchers in Taiwan to follow-up on all the medical services of these million individuals beginning with the initiation of the Taiwan NHI program in 1995.

This study was exempt from full review by the Institutional Review Board (IRB) after consulting with the director of the Taipei Medical University IRB, since the LHID2000 consisted of anonymous secondary data released to the public for research purposes.

\section{Selection of cases and controls}

We identified 1416 incident patients pathologically diagnosed with KC (ICD-9-CM codes 189.0, 189.1, or 189.2) from the LHID2000 who received their diagnoses in ambulatory care visits or hospitalizations between January 2002 and December 2009. We excluded patients aged less than 40 years since the incidence rate of $\mathrm{KC}$ in this group was very low, and to avoid potentially contaminating the homogeneity of our cases and to avoid bias $(n=108)$. As a result, 1308 patients with $\mathrm{KC}$ were included in this study. We assigned their first diagnosis of $\mathrm{KC}$ as the index date.

In this study we selected 5 matched controls per case $(n=6540)$ from the remaining patients of the LHID2000, and in accordance with prior studies stratified by sex, age on a 10-year basis (40-49, 50-59, 60-69, 70-79, and >79), and index year to match the distributions in the cases. ${ }^{16}$ All selected controls were assured to have never received a diagnosis of KC prior to 2009 (the LHID2000 data available was from 1995 to 2009). For these controls, their first use of ambulatory care occurring during the index year was assigned as the index date.

\section{Outcomes measured}

This study compared the prevalence and risk of having previously been diagnosed with UC prior to the index date between cases and control. We identified UC patients based on a diagnosis of UC (ICD-9-CM codes 592 [calculus of the kidney and ureter], 592.0 [calculus of the kidney], 592.1 [calculus of the ureter] or 592.9 [urinary calculus, unspecified]). To increase the validity of UC diagnoses, we only selected UC patients if they had received 2 or more UC diagnoses prior to the index date, with at least one being made by a urologist. In Taiwan, the first diagnosis for a condition indicates that a clinician performed an assessment for the condition in question. The second diagnosis reflects the presence of the condition based on the outcome of the clinical assessment. In Taiwan, UC is diagnosed based on a review of the patient's medical history and one or more of several clinical tests. All patients suspected of UC are screened with urinalysis, which along with diagnostic imagining, helps in determining the differential diagnosis. Depending on the results, a suspected UC patient in Taiwan may undergo one or more of the following procedures to rule out other possible conditions and to provide more evidence for the presence of UC: plain-film radiography, ultrasound, intravenous pyelography, and noncontrast helical computed tomography.

\section{Statistical analysis}

In this study, all analyses were performed using the SAS system (SAS System for Windows, Version 8.2, SAS Institute Inc., Cary, NC). Chi-square tests were used to compare the differences in monthly income (New Taiwan dollar [NT\$]0NT15 840; NT15841-NT25 000; and 2NT25 001) and the geographic location (Northern, Central, Eastern, and Southern Taiwan) and urbanization level of the patient's residence (5 levels), as well as the distribution of comorbidities. In Taiwan, all 359 cities/towns were stratified into 5 urbanization level in accordance with a prior study, with 1 referring to the "most urbanized" and 5 referring to the "least urbanized." ${ }^{15}$ We intentionally matched the variable of urbanization level between cases and controls to assure that cases and controls were reasonably similar in terms of unmeasured neighbourhood socioeconomic characteristics. We selected and defined the comorbidities taken into consideration in our statistical modeling by ICD-9-CM codes. These comorbidities included hypertension (ICD-9CM codes 401-405, 642), diabetes (ICD-9-CM codes 250. and 648.0), renal disease (ICD-9-CM codes 582-586, 5900), obesity (ICD-9-CM code 278), and cystic kidney disease (ICD-9-CM codes 753.1 and 753.10). These comorbidities were included only if they were obtained before the index date. In addition, we further used conditional logistic regres- 
sion (conditioned on sex, age [10-year basis], and index year) to examine associations between $\mathrm{KC}$ and having been previously diagnosed with UC. All of the above mentioned variables were adjusted for in the regression models. In addition, we analyzed the association of KC with previously diagnosed UC stratified by sex. The conventional $p \leq 0.05$ was used to determine statistical significance.

\section{Results}

Of the total sample of 7848 subjects, the mean age was 65.3 years with a standard deviation of 12.3 years (Table 1). After matching for sex and age, we found a higher prevalence of renal disease $(23.9 \%$ vs. $4.9 \%, p<0.001)$ and cystic kidney disease $(0.6 \%$ vs. $0.1 \%, p<0.001)$ than in the control group. There were no significant differences in the prevalence of hypertension, diabetes, obesity, or tobacco use disorder between cases and controls. As for demographic characteristics, patients were more likely to reside in the southern part of Taiwan $(p=0.026)$ and in more urbanized communities $(p=0.046)$ than in the controls.

Of the total sample, $1262(16.1 \%)$ had been diagnosed with UC before the index date; UC was found in $415(31.7 \%)$ cases and in $847(13.0 \%)$ controls $(p<0.001)$ (Table 2$)$. After adjusting for monthly income, geographic location, urbanization level, hypertension, diabetes, renal disease, obesity, cystic kidney disease, tobacco use disorder, and alcohol abuse, we found that, upon conditional logistic regression analysis (conditioned on sex, age, and index year), patients were more likely to have been previously diagnosed with UC before the index date when compared with controls (odds ratio [OR] 3.18; 95\% confidence interval [CI] 2.753.68; $p<0.001)$.

Table 2 also shows the association between KC and UC according to the type of kidney cancer. This study included 780 RCC patients and 528 TCC patients. We found that, after adjusting for monthly income, geographic location, urbanization level, hypertension, diabetes, renal disease, obesity, cystic kidney disease, tobacco use disorder, and alcohol abuse, the OR of having prior $U C$ for patients with RCC when compared with controls was $2.76(95 \% \mathrm{Cl} 2.31$ $3.29, p<0.001)$. Similarly, as compared to controls, the adjusted odds of prior $U C$ for patients with TCC were 3.96 (95\% Cl 3.23-4.86, $p<0.001)$.

The adjusted OR of prior UC was 2.93 (95\% Cl 2.42-3.55, $p<0.001)$ times more for male cases than those for male controls (Table 3). Among the female subjects, the conditional logistic regression analysis revealed that cases were more likely than controls to have UC before the index date (OR 3.59; 95\% Cl 2.87-4.48; $p<0.001$ ) after adjusting for monthly income, geographic location, urbanization level, hypertension, diabetes, renal disease, obesity, cystic kidney disease, and tobacco use disorder, and alcohol abuse.

\section{Discussion}

In this study, 1308 subjects were analyzed. Of these subjects, 780 suffered from RCC, 528 were diagnosed with TCC, and 6540 were controls. After adjusting for age, gender, comorbid medical disorders (hypertension, diabetes, cystic renal disease, and chronic kidney disease), and obesity, we found that the combined KC patients had a 3.18-fold higher risk of having been previously diagnosed with UC. We further analyzed this association based on the type of cancer and found TCC and RCC patients were 3.96 and 2.76 times more likely to have been previously diagnosed with $U C$, respectively, compared to controls. While in the case of TCC, the results of this study are merely confirmatory, the association detected between RCC and UC refutes the results of the most powerful study conducted concerning this association to date.

The associations detected in this study between UC and TCC are consistent with clinical reports demonstrating a link between UC and TCC. ${ }^{17}$ Previous studies have speculated that the mechanisms underpinning this association maybe involve the chronic irritation and infections related to UC. ${ }^{18}$ The irritation and infections would cause inflammatory cells, (including neutrophils, macrophages, dendritic cells, mast cells) and lymphocytes at the damage site. The lymphocytes would then secrete a large number of cytokines and chemokines which promote the growth of neoplastic cells and contribute to the onset and progression of cancer. ${ }^{19}$

Stones have furthermore been demonstrated in animal studies to cause neoplastic changes in the adjacent urothelium. ${ }^{20,21}$ Therefore, it is possible that the association in this study between UC and KC resulted from UC-attributed irritation and infections which promoted proliferative urothelial changes and cancer. ${ }^{5}$ In addition, the same inflammatory cytokines may also regulate the progression and metastasis of tumours.

Chow and colleagues further show that most of the renal pelvis/ureter cancers occur on the same side as the stone, and that the highest risk for renal pelvis/ureter cancers was found among patients with associated infections. ${ }^{5}$

While the results of this study regarding the association between RCC and UC concur with previous studies, ${ }^{10,12}$ they stand in contrast to those detected by the largest study conducted concerning this association to date. ${ }^{5}$ Results obtained by Maclure and colleagues concur with our results. They investigated patients with a history of kidney stones and renal adenocarcinoma. They found renal adenocarcinoma patients to be $2.3(95 \% \mathrm{Cl} 1.3-4.1)$ times more likely than 605 neighbourhood controls to have had a history of kidney stones. ${ }^{10}$ Another previous study investigating the association between RCC and kidney stones was performed in northern Italy. In their hospital-based case-control study conducted between 1986 and 1989 on 240 patients with RCC and 665 


\begin{tabular}{|c|c|c|c|c|c|}
\hline \multirow{2}{*}{ Variable } & \multicolumn{2}{|c|}{ Patients with kidney cancer $(n=1308)$} & \multicolumn{2}{|c|}{ Controls $(n=6540)$} & \multirow{2}{*}{$p$ value } \\
\hline & Total No. & $\%$ & Total No. & $\%$ & \\
\hline Sex & & & & & 1.000 \\
\hline Male & 682 & 52.1 & 3410 & 52.1 & \\
\hline Female & 626 & 47.9 & 3130 & 47.9 & \\
\hline Age (years) & & & & & 1.000 \\
\hline $40-49$ & 171 & 13.1 & 855 & 13.1 & \\
\hline $50-59$ & 255 & 19.5 & 1275 & 19.5 & \\
\hline $60-69$ & 324 & 24.8 & 1620 & 24.8 & \\
\hline $70-79$ & 388 & 29.6 & 1940 & 29.6 & \\
\hline$>79$ & 170 & 13.0 & 850 & 13.0 & \\
\hline Monthly income & & & & & 0.056 \\
\hline No income & 248 & 19.0 & 1080 & 16.5 & \\
\hline NT\$1-15 840 & 200 & 15.3 & 911 & 13.9 & \\
\hline NT\$15 84125,000 & 588 & 44.9 & 3106 & 47.5 & \\
\hline$\geq \mathrm{NT} \$ 25,001$ & 272 & 20.8 & 1443 & 22.1 & \\
\hline Geographic region & & & & & 0.026 \\
\hline Northern & 623 & 47.6 & 3043 & 46.5 & \\
\hline Central & 263 & 20.1 & 1543 & 23.6 & \\
\hline Southern & 395 & 30.2 & 1799 & 27.5 & \\
\hline Eastern & 27 & 2.1 & 155 & 2.4 & \\
\hline Urbanization level & & & & & 0.046 \\
\hline 1 (most urbanized) & 416 & 33.6 & 1903 & 30.8 & \\
\hline 2 & 388 & 31.3 & 1817 & 29.4 & \\
\hline 3 & 174 & 14.0 & 1019 & 16.5 & \\
\hline 4 & 174 & 14.0 & 954 & 15.4 & \\
\hline 5 (least urbanized) & 88 & 7.1 & 485 & 7.9 & \\
\hline Chronic renal disease & 312 & 23.9 & 318 & 4.9 & $<0.001$ \\
\hline Diabetes & 274 & 21.0 & 1233 & 18.9 & 0.079 \\
\hline Hypertension & 609 & 46.6 & 2913 & 44.5 & 0.180 \\
\hline Obesity & 3 & 0.2 & 32 & 0.5 & 0.198 \\
\hline Cystic kidney disease & 8 & 0.6 & 9 & 0.1 & $<0.001$ \\
\hline Tobacco use disorder & 80 & 6.1 & 222 & 3.4 & $<0.001$ \\
\hline Alcohol abuse & 15 & 1.0 & 66 & 1.0 & 0.998 \\
\hline
\end{tabular}

NT: New Taiwan dollar.

controls, Talamini and colleagues found RCC patients had a weak positive association with prior kidney stones (OR 2.00 95\% Cl 1.07-3.73). ${ }^{11}$ Additionally, in a large international case-control study, it was found that the relative risks (RR) for renal cancer were significantlv increased bv a historv of kidney stones, after adjusting for the effects of age, gender, body-mass index, tobacco smoking, and study centre. ${ }^{12}$

These results were all refuted by a study conducted by Chow and colleagues on data drawn from the national Swedish In-patient Register and the National Swedish

Table 2. Prevalence and odds ratios for urinary calculi among the sampled patients

\begin{tabular}{|c|c|c|c|c|c|c|c|c|}
\hline \multirow{4}{*}{$\begin{array}{l}\text { Presence of prior urinary } \\
\text { calculi } \\
\text { Yes }\end{array}$} & \multirow{2}{*}{\multicolumn{2}{|c|}{ Control $(n=6540)$}} & \multirow{2}{*}{\multicolumn{2}{|c|}{ Patients with KC ( $n=1308)$}} & \multicolumn{4}{|c|}{ Patients with KC (n=1308) } \\
\hline & & & & & \multicolumn{2}{|c|}{$\operatorname{RCC}(n=780)$} & \multicolumn{2}{|c|}{$\operatorname{TCC}(n=528)$} \\
\hline & \multicolumn{2}{|c|}{$n, \%$} & \multicolumn{2}{|c|}{$n, \%$} & \multicolumn{2}{|c|}{$\mathrm{n}, \%$} & \multicolumn{2}{|c|}{$\mathrm{n}, \%$} \\
\hline & 847 & 13.0 & 415 & 31.7 & 229 & 29.4 & 186 & 35.2 \\
\hline No & 5693 & 87.0 & 893 & 68.3 & 551 & 70.6 & 342 & 64.8 \\
\hline Crude OR (95\% Cl) & \multicolumn{2}{|c|}{1.00} & \multicolumn{2}{|c|}{$3.17 * * *(2.76-3.64)$} & \multicolumn{2}{|c|}{$2.79 * * *(2.35-3.31)$} & \multicolumn{2}{|c|}{$3.79 * * *(3.12-4.60)$} \\
\hline Adjusted OR a $(95 \% \mathrm{Cl})$ & \multicolumn{2}{|c|}{1.00} & \multicolumn{2}{|c|}{$3.18 * * *(2.75-3.68)$} & \multicolumn{2}{|c|}{$2.76 * * *(2.31-3.29)$} & \multicolumn{2}{|c|}{$3.96 * * *(3.23-4.86)$} \\
\hline
\end{tabular}




\begin{tabular}{llcccc}
\hline Table 3. Odds ratios for urinary calculi among patients with kidney cancer and controls, by sex \\
\hline
\end{tabular}

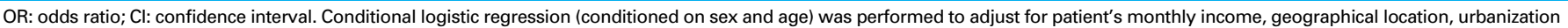
level, hypertension, diabetes, renal disease, obesity, cystic kidney disease, tobacco use disorder, and alcohol abuse; ${ }^{* *}$ indicates $p<0.001$.

Cancer Registry. In this study, the authors followed 61144 patients for 25 years who were hospitalized for kidney or ureter stones from 1965 to 1983 , but failed to detect an increased risk of RCC among UC patients. ${ }^{5}$ The present study found RCC patients to be 2.76 times more likely to have had a previous diagnosis of $U C$, thus adding support for the presence of a positive association between RCC and UC.

In this study we demonstrated that the association was much more prominent in females. One reason for the greater risk among females may involve their higher frequency of urinary tract infections. ${ }^{22}$ Another possibility involves the use of Chinese herbs. Several researchers have proposed that the use of Chinese herbs may have an important role in female TCC of the kidney and ureter. ${ }^{23,24}$ In a Taiwan-specific cohort of 6548 Chinese herbalists, female herbalists were found to have a higher risk of urological cancers than males. ${ }^{25}$

This study has limitations. The major limitation of this study is the lack of information regarding potential confounding factors, such as family history of $\mathrm{KC}$, cigarette smoking, body mass index, and occupational exposures, all of which may have contributed to the development of KC. By using ICD-9-CM coding, we were able to adjust for obesity, but it is possible that this may have resulted in an underestimation of the prevalence of obesity. In any case, the prevalence detected in this study was not much different from prior studies using the same database. ${ }^{26,27} \mathrm{We}$ also did not have access to any information regarding any protective factors, such as vegetable and fruit consumption, and therefore could not adjust for their effects. This may have been a confounding factor in the association between $\mathrm{KC}$ and $\mathrm{UC}$, as $\mathrm{UC}$ has been linked to dietary factors, and thus diet is related to both the exposure (UC) and outcome (KC). Our inability to account for these factors was due to the absence of this information in the administrative dataset analyzed in this study.

Secondly, we did not identify the stone site to confirm whether it was in the same location as KC, as it was not part of the data. Although the information contained in the LHID2000 is derived from medical records, not all the information present in the medical records is carried over into the LHID200 as it is primarily used as an administrative database for medical claims. Nevertheless, in our nationwide population-based analysis, we have collected conventional risk factors and comorbidities at baseline, and $\mathrm{KC}$ remained a significant risk factor after adjusting for these confounding factors.

Thirdly, the diagnoses used in this study were sourced from an administrative database, and therefore, they may be less accurate than diagnoses undertaken individually through standardized imaging reports in a prospective study design. Moreover, while a diagnostic validation study was not conducted for the specific diagnoses used in this study for the cases registered in the $\mathrm{NHI}$, many prior studies have demonstrated the high validity of the LHID2000, ${ }^{14,26}$ and hundreds of papers employing the LHID2000 have been published in internationally peer-reviewed journals. Furthermore, the $\mathrm{NHI}$ Bureau implements routine sample cross-checks of the claims of every hospital and clinic claims with its medical charts and enforces putative measures for coding infractions. Therefore, it is generally believed that the NHI's checks and balances foster accurate coding.

Fourthly, this study may have been vulnerable to a surveillance bias as patients with $U C$ would have been more likely to be screened for cancer. Furthermore, we were not able to assess this possibility by analyzing the elapsed time between the exposure of interest and the onset of disease to compare with the control group. As the $\mathrm{NHI}$ program was only initiated in 1995, we could only trace the subjects analyzed in this study back to that date. Therefore, it is likely that many of the UC patients analyzed in this study were not new cases, and therefore any analysis of the interval between the first diagnosis of UC and the diagnosis of $\mathrm{KC}$ in this study would not be a valid indicator for the elapsed time between the exposure of interest and the onset of disease.

Finally, over 98\% of Taiwan's residents are of Chinese Han ethnicity. While the homogenous population may exempt our study from potential confounding by race, it also limits the generalizability to other ethnic groups. Therefore, the results found in this study may not be generally applied.

\section{Conclusion}

This study leveraged the data from the Taiwan LHID2000 to succeed in identifying the association between $\mathrm{KC}$, includ- 
ing RCC and TCC, and patients with a previous diagnosis of UC. These results highlight a need for urologists dealing with UC patients to monitor the potential risk for the subsequent development of KC.

Competing interests: Dr. Chung, Dr. Liu and Dr. Lin all declare no competing financial or personal interests.

This paper has been peer-reviewed.

\section{References}

1. Goldfarb DS. Increasing prevalence of kidney stones in the United States. Kidney Int 2003;63:1951-2. http://dx.doi.org/10.1046/i.1523-1755.2003.00942.x

2. Stamatelou KK, Francis ME, Jones $C A$, et al. Time trends in reported prevalence of kidney stones in the United States: 1976-1994. Kidney Int 2003;63:1817-23. http://dx.doi.org/10.1046/j.15231755.2003.00917.x

3. López M, Hoppe B. History, epidemiology and regional diversities of urolithiasis. Pediatr Nephrol 2010;25:49-59. http://dx.doi.org/10.1007/s00467-008-0960-5

4. Long LO, Park S. Update on nephrolithiasis management. Minerva Urol Nefrol 2007;59:317-25.

5. Chow WH, Lindblad $P$, Gridley $G$, et al. Risk of urinary tract cancers following kidney or ureter stones. J Natl Cancer Inst 1997;89:1453-7. http://dx.doi.org/10.1093/inci/89.19.1453

6. Ross RK, Paganini-Hill A, Landolph J, et al. Analgesics, cigarette, smoking, and other risk factors for cancer of the renal pelvis and ureter. Cancer Res 1989;49:1045-8.

7. McCredie M, Stewart JH. Risk factors for kidney cancer in New South Wales Australia. II. Urologic disease, hypertension, obesity, and hormonal factors. Cancer Causes Control 1992;3:323-31. http://dx.doi. org/10.1007/BF00146885

8. Liaw KL, Linet MS, McLaughlin JK, et al. Possible relation between hypertension and cancers of the renal pelvis and ureter. Int J Cancer 1997;70:265-8. http://dx.doi.org/10.1002/(SICI) 10970215(19970127)70:3<265::AID-IJC3>3.0.C0;2-V

9. McLaughlin JK, Mandel JS, Blot WJ. A population based case-control study of renal cell carcinoma. J Natl Cancer Inst 1984;72:275-84.

10. Maclure $M$, Willett W. A case-control study of diet and risk of renal adenocarcinoma. Epidemiology 1990;1:430-40. http://dx.doi.org/10.1097/00001648-199011000-00004

11. Talamini R, Barón AE, Barra $S$, et al. A case-control study of risk factor for renal cell cancer in northern Italy. Cancer Causes Control 1990;1:125-31. http://dx.doi.org/10.1007/BF00053163

12. Schlehofer $B$, Pommer W, Mellemgaard A, et al. International Renal-cell-cancer study. VI. The role of medical and family history. Int J Cancer 1996;66:723-26. hittp://dx.doi.org/10.1002/(SICI) 1097 0215(19960611)66:6<723::AID-IJC2>3.0.C0;2-1
13. Gau CS, Chang IS, Lin Wu FL, et al. Usage of the claim database of national health insurance programme for analysis of cisapride-erythromycin co-medication in Taiwan. Pharmacoepidemiol Drug Saf 2007;16:8695. http://dx.doi.org/10.1002/pds.1324

14. Cheng CL, Kao YH, Lin SJ, et al. Validation of the National Health Insurance Research Database with ischemic stroke cases in Taiwan. Pharmacoepidemiol Drug Saf 2011;20:236-42. http://dx.doi. org/10.1002/pds.2087

15. Kang JH, Chen YH, Lin HC. Comorbidity profiles among patients with ankylosing spondylitis: a nationwide population-based study. Ann Rheum Dis 2010;69:1165-8. http://dx.doi.org/10.1136/ ard.2009.116178

16. Chung SD, Keller JJ, Lin HC. Increased risk of depressive disorder within 1 year after diagnosis with urinary calculi in Taiwan. Psychiatry Res 2012;198:332-3. http://dx.doi.org/10.1016/i.psychres.2012.01.007

17. DeVita VT, Hellman S, Rosenberg SA. Cancer of the kidney and ureter. In: DeVita VT, Hellman S, Rosenberg SA, editors. Cancer: principles and practice of oncology. 5th ed. Philadelphia: Lippincott-Raven Publishers; 1997;1271-300.

18. Li WM, Chou YH, Li CC, et al. Association of body mass index and urine pH in patients with urolithiasis. Urol Res 2009;37:193-6. htrp://dx.doi.org/10.1007/s00240-009-0194-4

19. Federico $\mathrm{A}$, Morgillo $\mathrm{F}$, Tuccillo $\mathrm{C}$, et al Chronic inflammation and oxidative stress in human carcinogenesis. Int J Cancer 2007;121:2381-6. http://dx.doi.org/10.1002/iic.23192

20. Okumura $M$, Hasegawa $R$, Shirai $T$, et al. Relationship between calculus formation and carcinogenesis in the urinary bladder of rats administered the nongenotoxic agents thymine or melamine. Carcinogenesis 1992;13:1043-5. http://dx.doi.org/10.1093/carcin/13.6.1043

21. Ogasawara H, Imaida K, Ishiwata $\mathrm{H}$, et al. Urinary bladder carcinogenesis induced by melamine in F344 male rats: correlation between carcinogenicity and urolith formation. Carcinogenesis 1995;16:2773-7. http://dx.doi.org/10.1093/carcin/16.11.2773

22. Yoshikawa TT, Nicolle LE, Norman DC. Management of complicated urinary tract infection in older patients. J Am Geriatr Soc 1996;44:1235-41.

23. Hung PH, Shen $\mathrm{CH}$, Tsai HB, et al. Gender effect on renal outcome in patients with urothelial carcinoma. World J Urol 2011;29:511-6. http://dx.doi.org/10.1007/s00345-011-0682-3

24. Chang $\mathrm{CH}$, Yang $\mathrm{CM}$, Yang AH. Renal diagnosis of chronic hemodialysis with urinary tract transitional cell carcinoma in Taiwan. Cancer 2007;109:1487-92. http://dx.doi.org/10.1002/cncr.22557

25. Yang HY, Wang JD, LO TC, et al. Increased risks of upper tract urothelial carcinoma in male and female chinese herbalists. J Formos Med Assoc 2011;110:161-8. http://dx.doi.org/10.1016/S09296646(11)60026-0

26. Kang JH, Chen YH, Lin HC. Comorbidity profiles among patients with ankylosing spondylitis: a nationwide population-based study. Ann Rheum Dis 2010;69:1165-8. http://dx.doi.org/10.1136/ ard.2009.116178

27. Kang JH, Keller JJ, Chen YK, et al. Association between obstructive sleep apnea and urinary calculi: a population-based case-control study. Urology 2012;79:340-5. http://dx.doi.org/10.1016/i.urology.2011.08.040

Correspondence: Dr. Herng-Ching Lin, School of Health Care Administration, Taipei Medical University, 250 Wu-Hsing St., Taipei 110, Taiwan; fax: 886-2-2378-9788; henry11111@tmu.edu.tw 